\title{
CENTROSYMMETRIC GRAPHS AND \\ A LOWER BOUND FOR GRAPH ENERGY OF FULLERENES
}

\author{
Gyula Y. Katona \\ Department of Computer Science and Information Theory \\ Budapest University of Technology and Economics \\ Budapest, Hungary \\ and \\ MTA-ELTE Numerical Analysis and Large Networks \\ Research Group, Budapest, Hungary \\ e-mail: gyula.katona@gmail.com \\ MoRTEZA FAghani \\ Department of Mathematics, Payam-e Noor University \\ Tehran, I. R. Iran \\ e-mail: m_faghani@pnu.ac.ir
}

AND

Ali Reza Ashrafi

Department of Pure Mathematics

Faculty of Mathematical Sciences

University of Kashan, Kashan 87317-51167, I. R. Iran

e-mail: ashrafi@kashanu.ac.ir

\begin{abstract}
The energy of a molecular graph $G$ is defined as the summation of the absolute values of the eigenvalues of adjacency matrix of a graph $G$. In this paper, an infinite class of fullerene graphs with $10 n$ vertices, $n \geq 2$, is considered. By proving centrosymmetricity of the adjacency matrix of these fullerene graphs, a lower bound for its energy is given. Our method is general and can be extended to other class of fullerene graphs.
\end{abstract}

Keywords: centrosymmetric matrix, fullerene graph, energy.

2010 Mathematics Subject Classification: 05C35, 05C50, 92E10. 


\section{REFERENCES}

[1] A. Cantoni and P. Buter, Eigenvalues and eigenvectors of symmetric centrosymmetric matrices, Linear Algebra Appl. 13 (1976) 275-288. doi:10.1016/0024-3795(76)90101-4

[2] D. Cvetković, M. Doob, I. Gutman and A. Torgašev, Recent Results in the Theory of Graph Spectra (North-Holland Publishing Co., Amsterdam, 1988).

[3] D. Cvetković, P. Rowlinson and S. Simić, An Introduction to the Theory of Graph Spectra (Cambridge University Press, Cambridge, 2010).

[4] P.W. Fowler and D.E. Manolopoulos, An Atlas of Fullerenes (Clarendom Press, Oxford, 1995).

[5] P.W. Fowler and W. Myrvold, Most fullerenes have no centrosymmetric labelling, MATCH Commun. Math. Comput. Chem. 71 (2014) 93-97.

[6] A. Graovac, O. Ori, M. Faghani and A.R. Ashrafi, Distance property of fullerenes, Iranian J. Math. Chem. 2 (2011) 99-107.

[7] I. Gutman, The energy of a graph, Ber. Math.-Statist. Sekt. Forsch. Graz 103 (1978) $1-22$.

[8] I. Gutman, Bounds for all graph energies, Chem. Phys. Lett. 528 (2012) 72-74.

[9] I. Gutman and B. Zhou, Laplacian energy of a graph, Linear Algebra Appl. 414 (2006) 29-37. doi:10.1016/j.laa.2005.09.008

[10] I. Gutman, S. Zare Firoozabadi, J.A. de la Peña and J. Rada, On the energy of regular graphs, MATCH Commun. Math. Comput. Chem. 57 (2007) 435-442.

[11] H. Hua, M. Faghani and A.R. Ashrafi, The Wiener and Wiener polarity indices of a class of fullerenes with exactly $12 n$ carbon atoms, MATCH Commun. Math. Comput. Chem. 71 (2014) 361-372.

[12] H.W. Kroto, J.R. Heath, S.C. O'Brien, R.F. Curl and R.E. Smalley, $C_{60}$ : buckminsterfullerene, Nature 318 (1985) 162-163. doi:10.1038/318162a0

[13] Z. Liu and H. Faßbender, Some properties of generalized K-centrosymmetric Hmatrices, J. Comput. Appl. Math. 215 (2008) 38-48. doi:10.1016/j.cam.2007.03.026

[14] Z.-Y. Liu, Some properties of centrosymmetric matrices, Appl. Math. Comput. 141 (2003) 297-306. doi:10.1016/S0096-3003(02)00254-0

[15] V. Nikiforov, The energy of graphs and matrices, J. Math. Anal. Appl. 326 (2007) 1472-1475. doi:10.1016/j.jmaa.2006.03.072

[16] O. Rojo and H. Rojo, Some results on symmetric circulant matrices and on symmetric centrosymmetric matrices, Linear Algebra Appl. 392 (2004) 211-233. doi:10.1016/j.laa.2004.06.013 
Received 9 April 2013

Revised 13 September 2013

Accepted 10 November 2013 